\title{
ORIGINAL ARTICLE \\ Synergistic anti-tumor effects between oncolytic vaccinia virus and paclitaxel are mediated by the IFN response and HMGB1
}

\author{
B Huang ${ }^{1}$, R Sikorski ${ }^{1}$, DH Kirn ${ }^{2}$ and SH Thorne ${ }^{1}$
}

Recent developments in the field of oncolytic or tumor-selective viruses have meant that the clinical applications of these agents are now being considered in more detail. Like most cancer therapies it is likely that they will be used primarily in combination with other therapeutics. Although several reports have shown that oncolytic viruses can synergize with chemotherapies within an infected cancer cell, it would be particularly important to determine whether factors released from infected cells could enhance the action of chemotherapies at a distance. Here, we demonstrate in vitro synergy between oncolytic vaccinia and taxanes. However, we also show, for the first time, that this synergy is at least partly due to the release of factors from the infected cells that are capable of sensitizing surrounding cells to chemotherapy. Several cellular factors were identified as being mediators of this bystander effect, including type I interferon released soon after infection and high-mobility group protein B1 (HMGB1) released after cell death. This represents the first description of these mechanisms for beneficial interactions between viral and traditional tumor therapies. These data may provide a direct basis for the design of clinical trials with agents currently in the clinic, as well as providing insight into the development of next generation viral vectors.

Gene Therapy (2011) 18, 164-172; doi:10.1038/gt.2010.121; published online 26 August 2010

Keywords: oncolytic virus; paclitaxel; HMGB1; interferon; synergy

\section{INTRODUCTION}

Recent advances in the field of oncolytic virotherapy, utilizing replication or tropism selective viruses for the treatment of cancer, ${ }^{1,2}$ have included promising clinical responses with several different viral agents and significant anti-tumor effects in a variety of pre-clinical models. ${ }^{3-6}$ One advantage of this approach is that viruses typically use distinct mechanisms of tumor cell killing relative to more traditional chemotherapy and radiotherapy approaches. ${ }^{2}$ In addition, a variety of targeting mechanisms mean that different tumor phenotypic properties can be targeted with different viral strains. ${ }^{1}$ This means that oncolytic viruses are likely to work effectively in combination with more traditional therapeutic approaches as they can be tailored to target different properties of the heterogeneous population of malignant cells within the tumor and to destroy these cells by multiple mechanisms. ${ }^{7-9}$ As a result, more cells within the tumor will be destroyed and resistance will be less likely to occur. Indeed, several reports have demonstrated how viral therapies can be effectively combined with chemotherapy, although some viral therapies appear to only be effective in the clinic when used in combination with chemotherapeutic treatments. ${ }^{10-13}$

Although some groups have described mechanisms through which different viral-chemotherapy combinations may result in synergy between the two platforms, these primarily focus on improved killing of already infected cells. For example, paclitaxel treatment has been shown to enhance the ability of an oncolytic adenovirus to replicate, through stabilization of microtubules increasing intracellular movement of the viral particles. ${ }^{14}$ Alternatively, the regulation of certain cellular genes by taxane treatment has been shown to enhance oncolytic Herpes Simplex Virus (HSV) replication ${ }^{15}$ while cell death induced by the chemotherapy has also been shown to allow the large virus to better penetrate the tumor. ${ }^{16}$ Although these observations are important, most oncolytic agents form acute infections, such that viral infection will lead to cell death, and so enhancing the replication cycle or sensitizing only the infected cells to chemotherapy may not be the most effective way to improve the overall therapeutic benefits.

Here, we looked to determine whether oncolytic vectors based on vaccinia virus also synergize with chemotherapies, especially paclitaxel (Taxol). Further studies looked to define the mechanisms mediating synergy, examining the effects of the cell cycle and on factors released from infected cells. We define several novel mechanisms mediating the interactions between oncolytic vaccinia and chemotherapy, which may be applied in the clinic to directly enhance the effects of these chemotherapies.

\section{RESULTS}

Paclitaxel synergizes with oncolytic vaccinia virus in vitro

A panel of tumor cell lines was tested in a cell viability assay following treatment with serial dilutions of paclitaxel, vaccinia strains (wild-type strain, WR (Western Reserve), or oncolytic strain, vvDD), or with combinations of therapeutics in different ratios. In each case the therapeutics were added $24 \mathrm{~h}$ apart, and with either the chemodrug or oncolytic virus added first. Cell viability was determined after $72 \mathrm{~h}$.

${ }^{1}$ Departments of Surgery and Immunology, Hillman Cancer Center, University of Pittsburgh Cancer Institute, University of Pittsburgh, Pittsburgh, PA, USA and ${ }^{2}$ Jennerex Biotherapeutics ULC, San Francisco, CA, USA

Correspondence: Dr SH Thorne, Departments of Surgery and Immunology, Hillman Cancer Center, University of Pittsburgh Cancer Institute, University of Pittsburgh, 5117 Centre Avenue, Pittsburgh, PA 15238, USA.

E-mail: ThorneSH@UPMC.edu

Received 15 April 2010; revised 9 June 2010; accepted 11 July 2010; published online 26 August 2010 
a
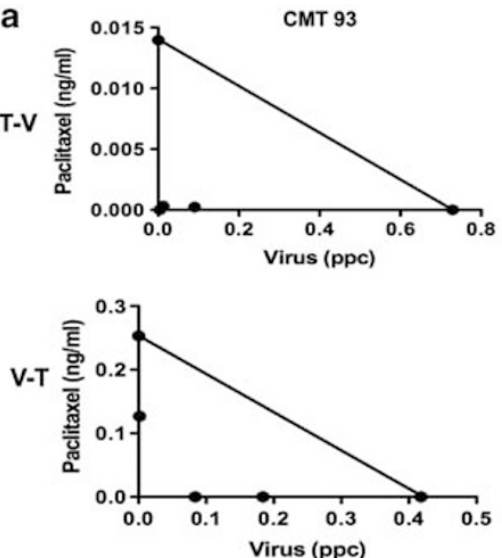
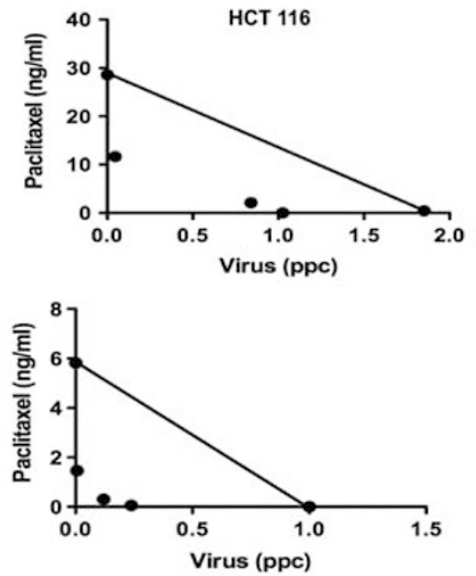
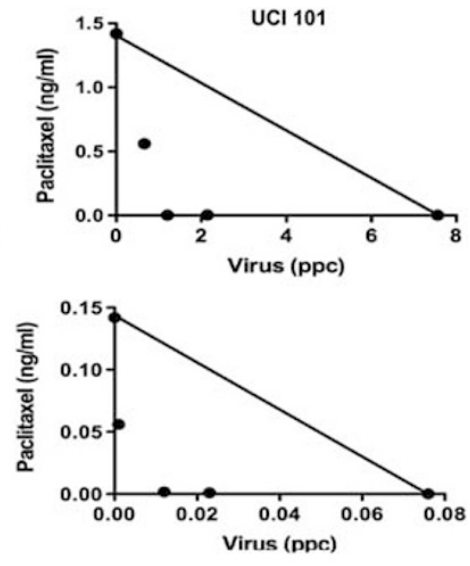
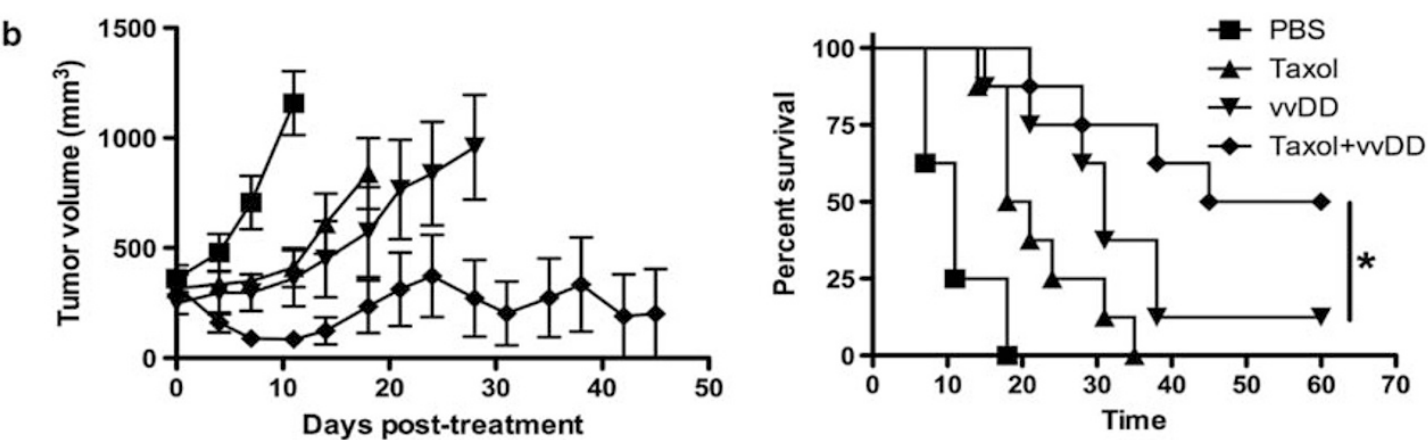

Figure 1 Vaccinia synergizes with paclitaxel. (a) Isobolograms from combination treatment of WR vaccinia and paclitaxel in murine and human tumor cell lines. Cells were infected with virus $24 \mathrm{~h}$ after (T-V) or before (V-T) paclitaxel at pre-determined concentrations and ratios (see Materials and methods). EC50 values for single therapies or different ratios of combinations were used to plot isobolograms. The straight line connects EC50 values for each agent alone and illustrates the expected result for additive effects. Data points below this line represent synergistic (combination indexes $(\mathrm{Cl}) \leqslant 0.8)$ and above the line are antagonistic $(\mathrm{Cl} \geqslant 1.2)$. All samples are run in triplicate, and graphs are representative of one of three repeat experiments. (b) Anti-tumor effects of combination therapy in vivo. Tumor growth for athymic nu-/nu- mice bearing palpable subcutaneous HCT-116 tumors after treatment with phosphate-buffered saline (PBS); vvDD-luc (intravenous delivered on day 1); paclitaxel $\left(60 \mathrm{mg} \mathrm{kg}^{-1}\right.$ delivered intraperitoneal on day 1,3 and 5 ); or both therapies, also shown is a survival curve, with mice killed when tumors reached $1400 \mathrm{~mm}^{3}, n=8$ per group. ${ }^{*} P=0.0052$.

This was repeated for a mouse colorectal cancer (CMT-93), a human colorectal (HCT-116) and a human ovarian cancer (UCI-101) cell line. The data produced were used to plot isobolagrams to determine whether the treatments synergized, had no enhancement, or produced antagonistic effects in the killing of tumor cells in vitro (Figure 1a), similar results were obtained with both WR and vvDD. It was found that for all three of the tumor cell lines tested, and for whichever order the therapies were used, synergy was produced. Similar experiments were attempted with a nontransformed cell line (NHeps, primary hepatocytes), but the relative resistance of these cells to both therapies made isobolagram construction impossible. Although combination therapy did produce significantly enhanced cell killing, this appeared to be less than additive (Supplementary Figure S1).

\section{In vivo therapeutic benefits of combining oncolytic vaccinia and} paclitaxel

In order to test whether this effect was also seen in vivo, mice (athymic nu-/nu-) bearing palpable HCT-116 tumors were treated with intraperitoneal injections of paclitaxel, intravenous injections of the oncolytic vaccinia strain vvDD-luc, or both drugs, or treated with phosphate-buffered saline as a control. It was seen that (Figure 1b), whereas vvDD or paclitaxel used as single agents slowed the growth of the tumors, they were generally not curative. However, 50\% (four of eight) of the animals treated with the combination resulted in complete and durable responses. There were also no overt signs of increased toxicity in the animals as a result of the combined therapy.

\section{Paclitaxel reduces overall viral replication, but not enveloped virus} (EV) production

It is possible that, because paclitaxel and oncolytic vaccinia synergized in their tumor cell killing in vitro, the infected cells may be destroyed more rapidly, so limiting viral replication. This may represent a longterm disadvantage to such combination therapies. Initially, examination of viral gene expression (bioluminescence) from vvDD-luciferase infected HCT-116 cells alone or in combination with paclitaxel determined that viral gene expression was greatly reduced when virus was used in combination with chemotherapy, with a $\log$ reduction in signal $24 \mathrm{~h}$ post-infection (Figure 2a). This increased even further at $48 \mathrm{~h}$ post-infection, but at this time there was extensive cell death in the combination treated wells. Plaque assay of samples taken $48 \mathrm{~h}$ post-infection confirmed that the combination with paclitaxel greatly reduced overall viral replication in a one-step growth curve (Figure $2 \mathrm{~b}$ ). However, vaccinia is produced in several different forms during the infection cycle, including an EV form, that is, produced and released from infected cells at the earliest times after infection. ${ }^{17}$ This form of the virus, which typically represents $<10 \%$ of the overall virus produced is therefore believed to be of critical importance for spread both within a tissue and within a host 


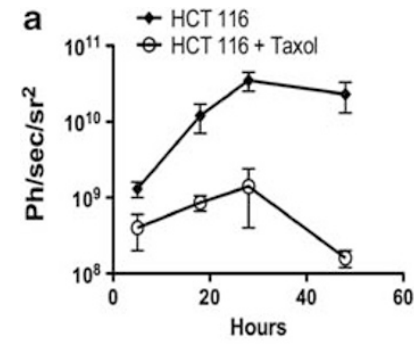

b
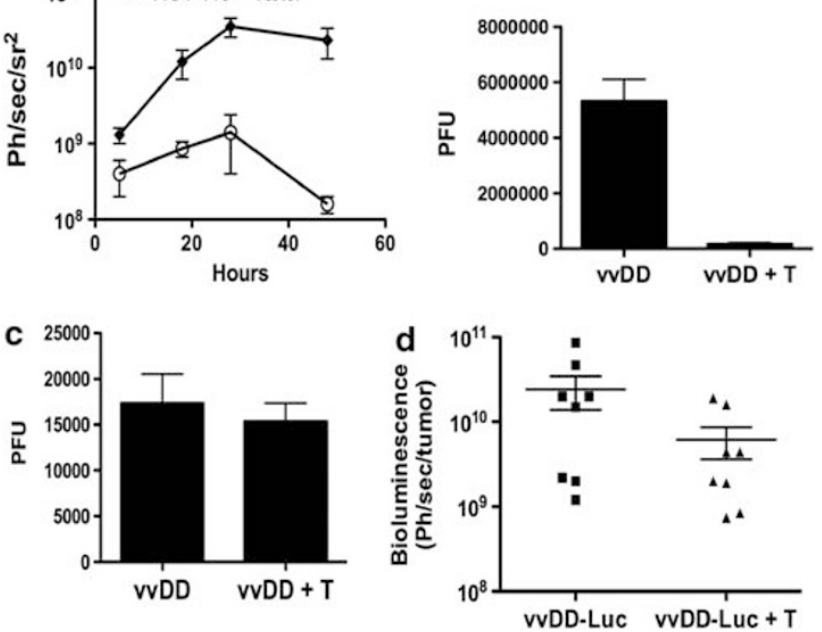

Figure 2 Paclitaxel reduces vaccinia replication (a) viral gene expression from vvDD-luc (MOI 1.0) infected HCT-116 cells with or without paclitaxel $\left(6 \mathrm{ng} \mathrm{ml}^{-1}\right.$ ) present (as determined by bioluminescence imaging; BLI) measured at times after viral infection average of three replicates $(P<0.001$; luciferase expression was driven from the pSE/L, synthetic early and late promoter). (b) Total viral production $48 \mathrm{~h}$ after infection as determined by plaque assay $48 \mathrm{~h}$ after infection $(P<0.05)$. (c) Production of the enveloped virus (EV) form of vaccinia, as determined by plaque assay from media above infected cells at $12 \mathrm{~h}$ post-infection and after exposure to anti-IMV neutralizing antibody. (d) Mice as in Figure $1 \mathrm{~b}$ treated with vvDD-luc were imaged (BLI) at 7 day after delivery of virus, and viral gene expression from within the tumor determined $(n=8)$, differences were not significant $(P=0.11)$.

organism. The other, intracellular mature virus (MV) form of the virus is not released until after the cell is lysed. We therefore wished to determine whether the reduction in viral replication seen in combination with paclitaxel was due to reduction in the levels of MV, EV or both. We titered the levels of virus released into the media at early times (12 h) after infection subsequent to exposure to MV-neutralizing antibody (Figure 2c), and it was seen that no significant reduction in the levels of EV were seen. This indicates that the reduction in overall viral replication was due to premature lysis of the cell leading to lower $\mathrm{MV}$ production. The normal production of EV may allow for unchanged early spread of the virus within the tumor. This was confirmed in vivo, with the level of gene expression being measured from vvDD-luc within HCT-116 tumors with and without paclitaxel (experiment as in Figure 1b). It was found that at 7 days after viral treatment, there was only a small but nonsignificant reduction in the level of gene expression when paclitaxel was also applied (Figure 2d).

\section{Paclitaxel effects on the cell cycle enhance vaccinia infection}

It is known that vaccinia preferentially replicates in cells in the G2/M or S-phase of the cell cycle, and induces proliferation (and an increase in the percentage of cell in these phases) following infection. ${ }^{18}$ It is also known that taxanes block the cell cycle at the G2/M interphase, so producing an accumulation of cells in the S-phase and G2/M. ${ }^{19}$ We used a vaccinia strain with Green Fluorescent Protein (GFP) fused to the viral A5L protein, a DNA packaging protein that is one of the most abundant within the viral particle. ${ }^{20}$ As such, it is possible to detect single viral particles by fluorescence microscopy or flow cytometry. This virus was used along with 7-amino-actinomycin staining to assess the relative levels of initial infection and the position in the cell cycle of
HCT-116 cells alone or following paclitaxel treatment (as determined $2 \mathrm{~h}$ after addition of the virus, before the viral infection itself can affect the position of the cells in the cell cycle). It was seen (Figure 3) surprisingly that initial viral infection appeared almost exclusively in cells in the S or G2/M phase, even in untreated cells. Following $18 \mathrm{~h}$ of paxlitaxel treatment the vast majority of the cells are already in the G2/ $\mathrm{M}$ phase, and so might be more susceptible to viral infection. Indeed, it was confirmed that after $2 \mathrm{~h}$ of exposure to A5L-GFP expressing vaccinia virus (at the same MOI of 1.0), the percentage of cells infected was $3.2 \%$ for untreated cells, but had almost doubled to $6.1 \%$ for paclitaxel treated cells. A second drug known to block cell cycle at G2/ $\mathrm{M}$ was also applied (Vincristine), and again it was seen that the accumulation of cells in the G2/M phase led to a marked increase in the number of cells infected with virus.

\section{Spent media from vvDD-infected cells can sensitize un-infected} cells to paclitaxel

The observation that paclitaxel pre-treatment can sensitize cells to vaccinia infection through accumulation of cells in the S-phase can explain why the combination therapies synergize when the chemotherapy is added first, but not when the virus is added first. We noticed that increased cell death subsequent to paclitaxel addition was observed even when very small levels of virus were present $(<0.01$ PFU per cell). This led us to examine the possibility that the virus was capable of producing a bystander effect, with uninfected cells becoming sensitized to paclitaxel treatment. As such, we took media from HCT-116 cells infected with vvDD for different lengths of time $(6,12$ and $24 \mathrm{~h}$ ) or control cells that had been left uninfected for the same lengths of time and filter sterilized these to remove any virus (this was first confirmed by plaque assay-Supplemental Figure 2). This spent media was added to fresh, uninfected HCT-116 cell layers in combination with paclitaxel (Figure 4a). It was seen that media collected at $6 \mathrm{~h}$ and at $24 \mathrm{~h}$ from infected cell layers were capable of enhancing the effects of paclitaxel mediated cell killing. Interestingly, media taken at $12 \mathrm{~h}$ post-infection did not lead to enhanced cell killing. This implied that different factors (one or more released early after infection, and one or more late) are each capable of enhancing the therapeutic effects of paclitaxel. This experiment was repeated in further cell lines in order to determine whether these effects were common (Figure $4 \mathrm{~b}$ ). As such, the human tumor cell lines UCI-101 and MCF-7 and the mouse tumor cell lines Lewis lung carcinoma and CMT-93 were examined. In all cases, the 'late effect' was seen (with enhanced paclitaxel effects seen in combination with media from cells infected for $24 \mathrm{~h}$ ). The 'early' enhancement with media from cells infected for only $6 \mathrm{~h}$ was seen in some (UCI-101; Lewis lung carcinoma), but not all (MCF-7; CMT-93) of the cell lines tested. In all cases, a similar pattern was seen, with the media taken at 6 h.p.i. leading to enhanced sensitivity to low doses of paclitaxel only (when the effect was present), whereas the media taken at 24 h.p.i. was capable of creating greater overall sensitivity to paclitaxel, and over a greater range of concentrations. Together these data supported the conclusion that two or more independent factors were mediating the increased sensitivity to paclitaxel.

\section{Interferon (IFN) release early after viral infection sensitizes cells to paclitaxel}

A possible factor that is likely to be released early after infection is type I IFN. We initially examined whether recombinant IFN could produce the same effects as media taken from infected cells 6 h.p.i (Figure 5a). This was confirmed to be the case. We therefore determined whether IFN was released from infected UCI-101 cells (that demonstrated the 


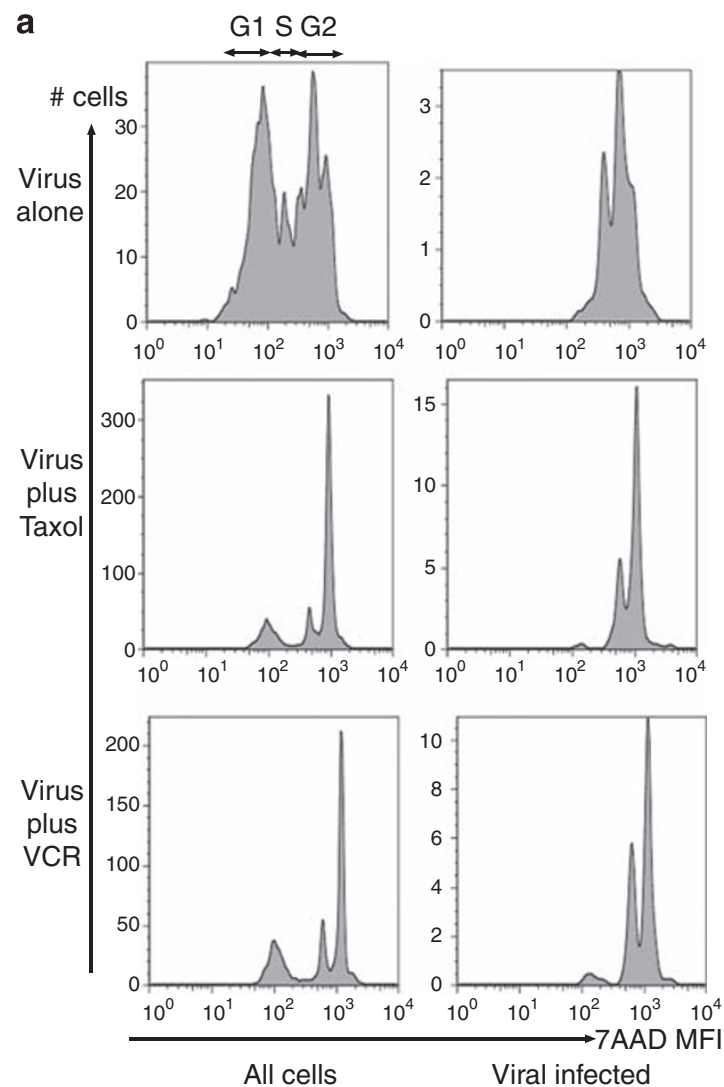

b
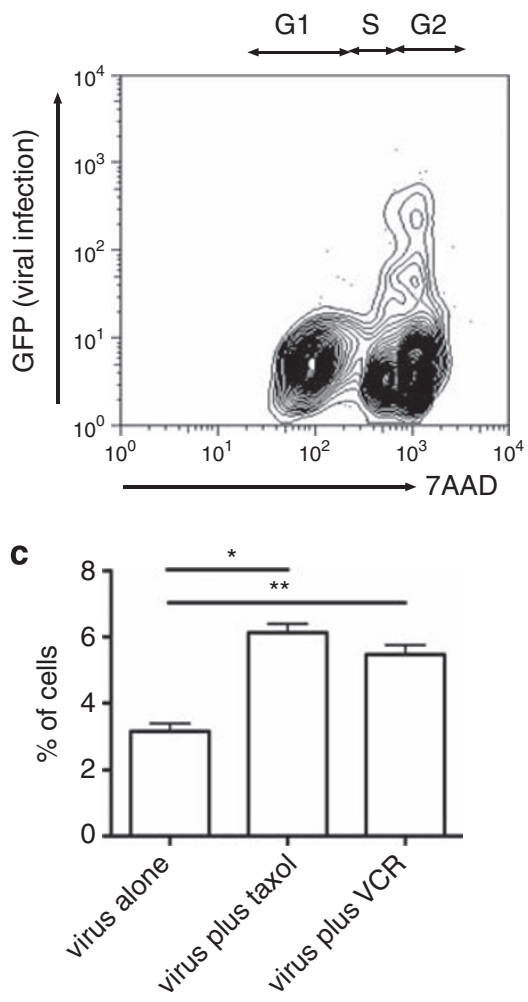

Figure 3 (a) HCT-116 cells were treated with phosphate-buffered saline, paclitaxel $\left(6 \mathrm{ng} \mathrm{ml}^{-1}\right.$ ) or vincristine (VCR at $40 \mathrm{ng} \mathrm{ml}^{-1}$ ) for $18 \mathrm{~h}$ before infection with WR strain carrying an A5L-GFP fusion gene for $2 \mathrm{~h}$. After this time 7-amino-actinomycin (7-AAD) staining was used to determine cell cycle status of the cells by flow cytometry ( $\mathrm{MFI}=$ mean fluorescence intensity). The percentage of GFP positive cells was determined, and the cell cycle status of the GFP positive cells shown. (b) HCT-116 cells treated with paclitaxel for $18 \mathrm{~h}$ and then A5L-GFP vaccinia virus for $6 \mathrm{~h}$ were stained with 7 -AAD for cell cycle analysis (as in (a), representative plot of 7-AAD against GFP is shown, to demonstrate how viral infection appears primarily in cells in the G2/M or S-phase. (c) Average values for three repeats of experiment as in (a). ${ }^{*} P=0.0011 ;{ }^{*} P=0.012$.

sensitizing effect with media taken 6 h.p.i.). As expected, enzymelinked immunosorbent assay (ELISA) showed that IFN was indeed produced by these cells. Interestingly, the kinetics of IFN release mirrored the sensitivity data, with a peak of IFN release at 6 h.p.i., which dropped off at later times (Figure 5b). Finally, in order to verify that IFN release was essential for this enhanced sensitivity to paclitaxel, IFN receptor blocking antibody was added to the filter sterilized media before addition to the second cell layer. It was confirmed that the addition of this antibody was sufficient to block the sensitizing effect of media taken at 6 h.p.i (Figure $5 \mathrm{c}$ ).

In order to determine why some cell lines (MCF-7 and CMT-93) did not demonstrate this early sensitization effect, the release of type I IFN from these cell lines was examined after vaccinia infection. It was found (Figure 5d) that MCF-7 was unable to produce IFN (explaining the lack of effect in this cell line). CMT-93, did produce IFN, but at significantly lower concentrations than the responsive cell lines HCT-116 or Lewis lung carcinoma. We therefore looked to determine wherther these cells were capable of responding to type I IFN (Figure 5e). It was found that these cells were not sensitized to paclitaxel killing by the addition of recombinant type I IFN. It is unclear what the defect in these cells is, whether it is a general lack of response to IFN, or if it is more specific to the effect on paclitaxel killing, but this does provide further support to the conclusion that type I IFN is mediating this early sensitizing effect.
HMGB1 released after viral-mediated cell death is a key mediator of enhanced sensitivity to paclitaxel

Because it is believed that the initially infected cells will begin to be destroyed because of viral infection at around 24 h.p.i., it was hypothesized that factors released by viral-mediated cell lysis may be responsible for enhancing sensitivity to paclitaxel at this time. Danger-associated molecular pathogen (DAMP) molecules are known to be released as a result of uncontrolled cell death and so might be produced by viral-mediated cell death. ${ }^{21}$ Probably the best-studied DAMP molecule is HMGB1. We therefore initially determined by ELISA whether HMGB1 was released. It was confirmed that vaccinia infection did lead to HMGB1 release (Figure 6a). A recombinant peptide representing the A-box region of HMGB1 and that is frequently used as an inhibitor or HMGB1 function ${ }^{22}$ was then used to determine whether HMGB1 was involved in mediating the sensitizing effect for media collected 24 h.p.i (Figure 6b). The addition of HMGB1 A-box was found to reduce the sensitizing effect of media collected 24 h.p.i., implying that this protein is an essential determinant or the key factor mediating this effect. Because the mechanism of action of HMGB1 A-box is not well defined, we also examined a second inhibitor of HMGB1, glycyrrhizin. It was found that addition of glycycrrhizin to spent media from an infected cell layer could partially block the ability of this media to sensitize a fresh cell layer to paclitaxel (Figure 6c). This supports the 
a

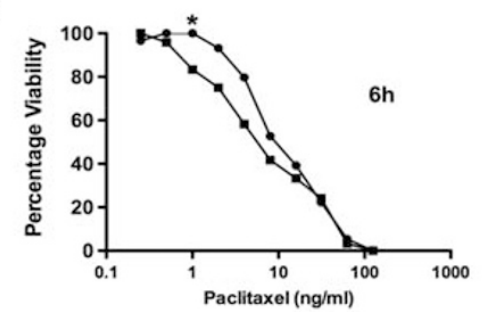

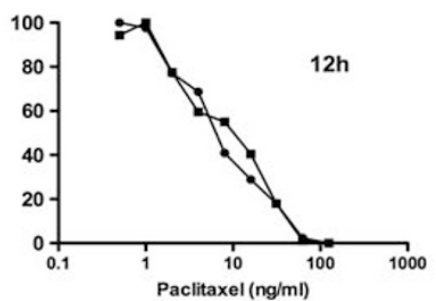

b
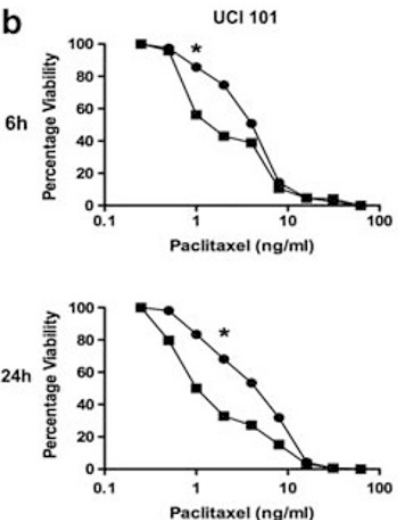

MCF-7
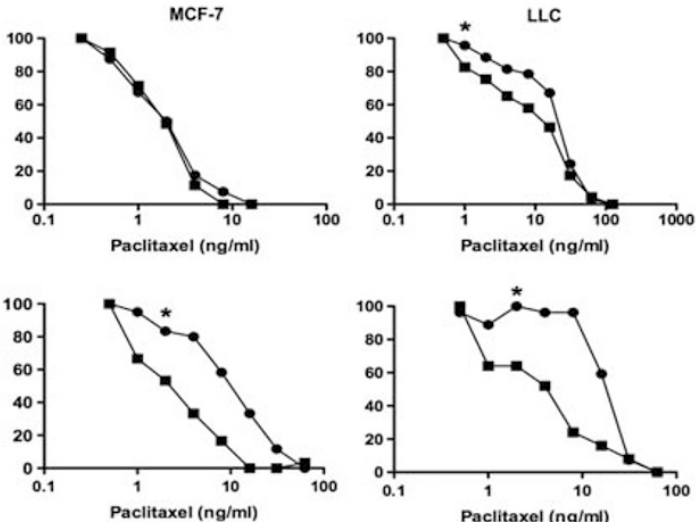
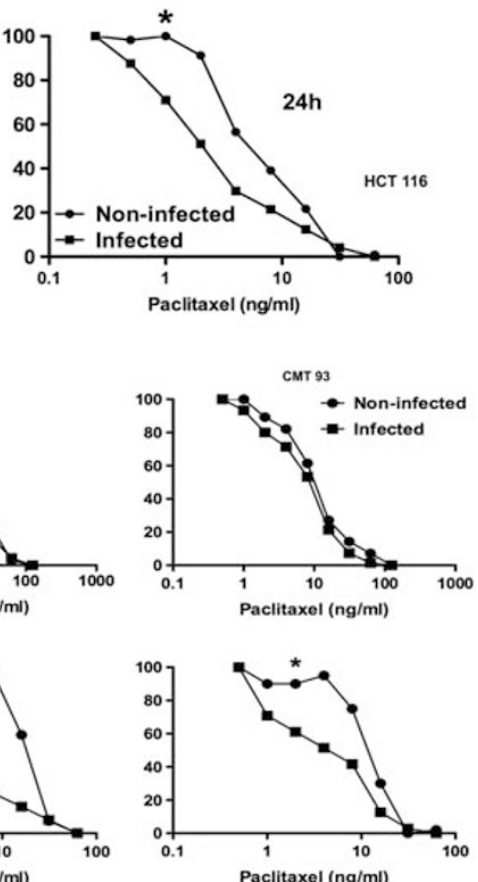

Figure 4 Indicated cell lines expressing luciferase were infected with vaccinia (vvDD; MOI 5.0) for the indicated times before supernatant was removed, filter sterilized and added to fresh cells of the same cell line (media from uninfected cells was used as a control). Serial dilutions of paclitaxel were added to these cells and survival measured by bioluminescence imaging (and confirmed by MTS assay, not shown) after $48 \mathrm{~h}$. Samples run in triplicate and graphs shown are representative of two independent experiments; ${ }^{*} P<0.05$. (a) HCT 116 (b) panel of human and murine tumor cell lines.
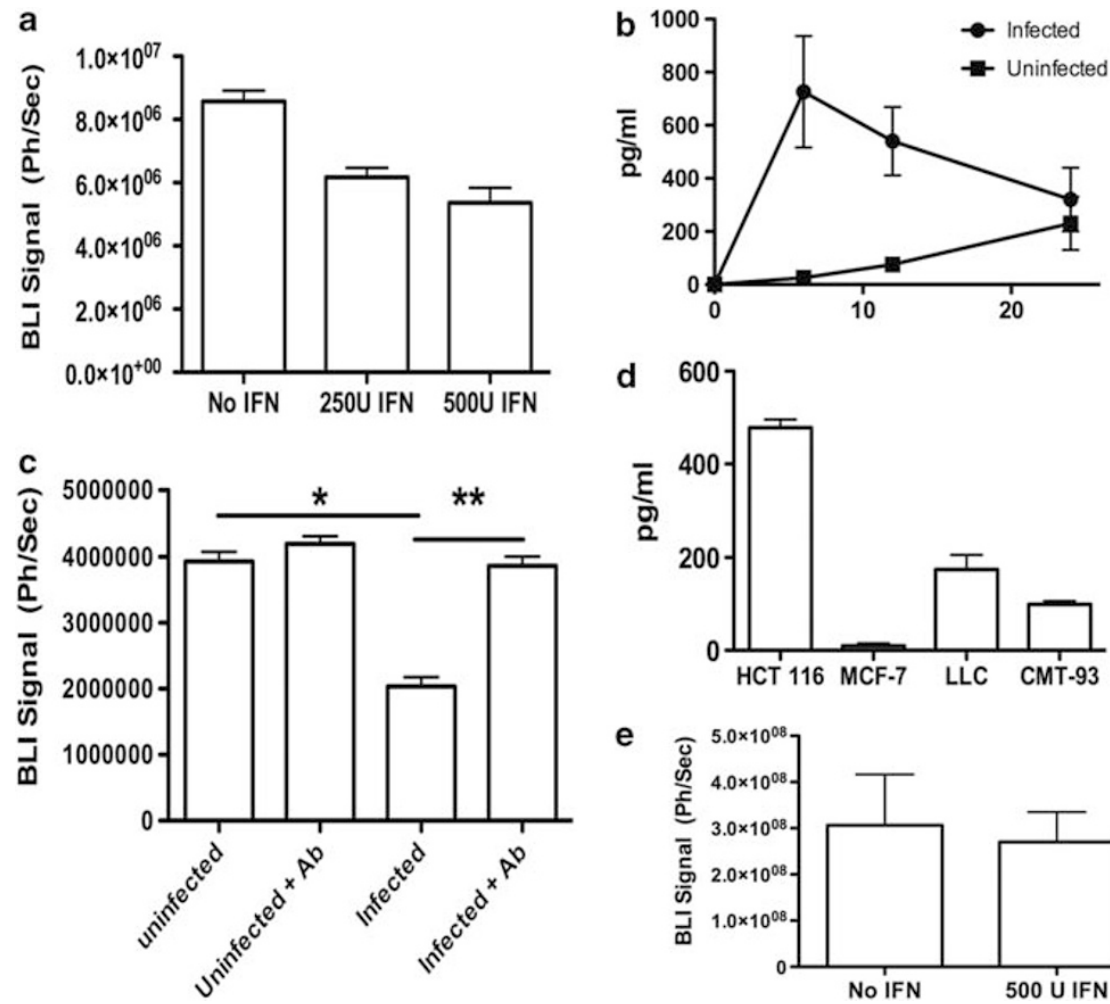

e

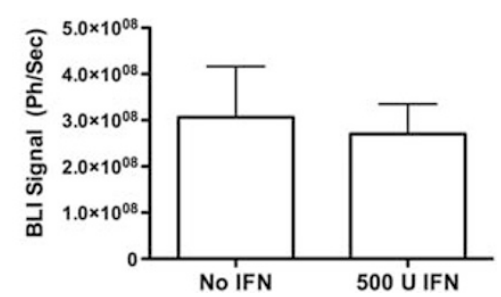

Figure 5 Type I IFN mediates enhanced sensitivity to paclitaxel. (a) Recombinant IFN- $\beta$ was added to UCl-101 cells along with paclitaxel and survival measured after $48 \mathrm{~h}$ by bioluminescence imaging (BLI). Survival was significantly reduced relative to no IFN ( $P=0.0063$ for $250 \mathrm{U}$ and 0.0054 for $500 \mathrm{U}$ IFN). (b) ELISA for IFN- $\beta$ production from vvDD infected or uninfected UCl-101 cells. (c) Effects of addition of anti-IFN $\alpha / \beta$ receptor blocking antibody or a control antibody on sensitizing of cells to paclitaxel with media taken at 6 h.p.i. ${ }^{*} P=0.0008$; ${ }^{* *} P=0.0009$. (d) Type I IFN production from different cell lines. Media was taken from above cell layers infected with vVDD (MOI 5.0, $6 \mathrm{~h}$ ) and ELISA for human or mouse IFN- $\beta$ according to manufacturer's instructions. (e) Effect of recombinant IFN- $\beta$ on CMT-93 cells along with paclitaxel. Survival measured after $48 \mathrm{~h}$ by BLI. 


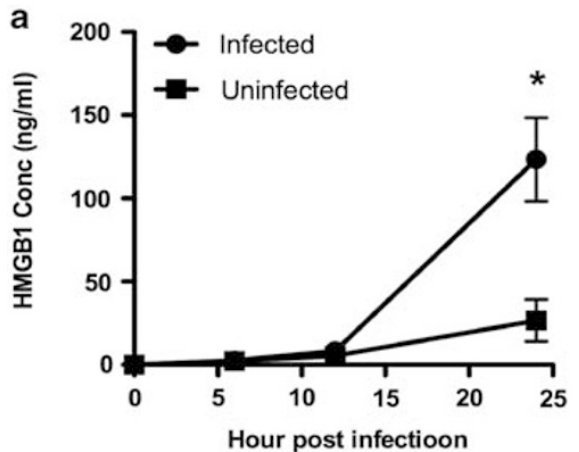

b

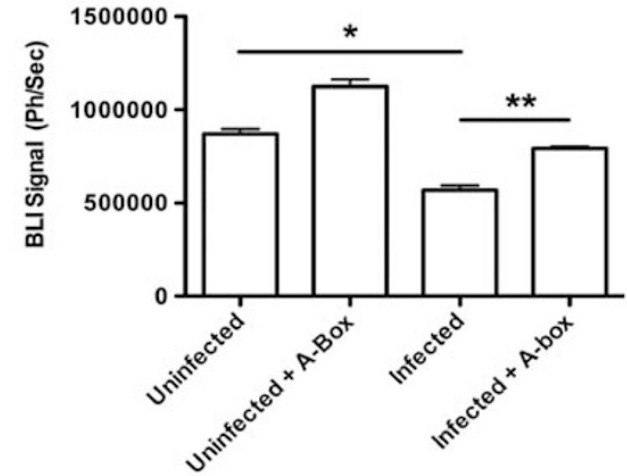

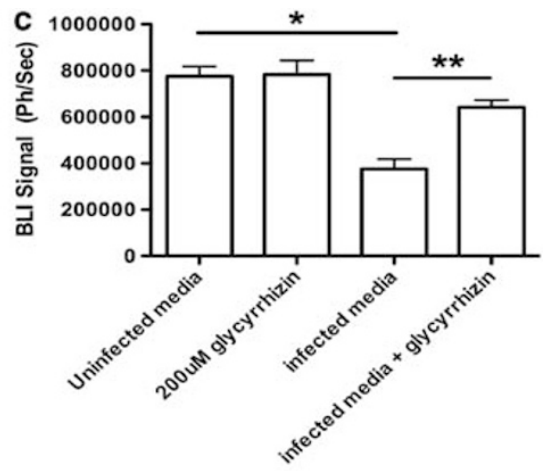

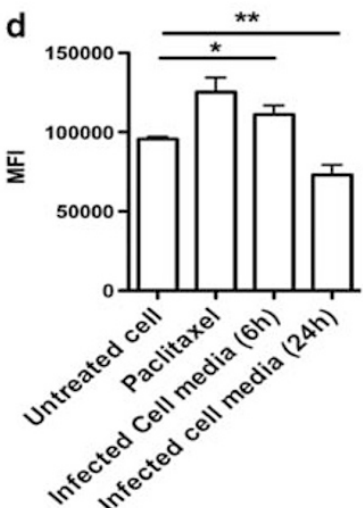

Figure 6 HMGB1 can enhance sensitivity of UCl-101 cells to paclitaxel. (a) Release of HMGB1 from infected (vvDD MOI 1.0) and uninfected UCl-101 cells as determined by ELISA, data run in triplicate $\left({ }^{*} P=0.0012\right)$. (b) Use of HMGB1 A-box as an inhibitor of HMGB1 function. HMGB1 A-box or PBS was added to media collected from infected or uninfected $\mathrm{UCl}-101$ cells taken at $24 \mathrm{~h}$ post-infection ( ${ }^{*} P=0.0012 ;{ }^{*} P=0.0013$ ). (c) Use of $200 \mu \mathrm{m}$ glycyrrhizin as an alternative inhibitor of HMGB1. Glycyrrhizin was added to media from infected or uninfected cells and the effect on the media's ability to sensitize a fresh $\mathrm{UCl}-101$ cell layer to paclitaxel $\left(6 \mathrm{ng} \mathrm{ml}^{-1}\right)$ was determined as before. ${ }^{*} P=0.0028 ;{ }^{*} P=0.0072$. (d) Caspase-3 assay performed on UCl-101 cells treated as indicated for $24 \mathrm{~h} ;{ }^{*} P=0.021 ; * * P=0.015$

hypothesis that HMGB1 was at least partially mediating the late sensitizing effect.

Because HMGB1 is known to be capable of inducing apoptosis, necrosis or autophagy depending on its oxidative state and the presence of co-factors, we looked to determine whether the media taken 24 h.p.i. was inducing apoptosis in fresh cells in a caspase-3 assay. As expected, paclitaxel, and also media taken 6 h.p.i (containing IFN) were both capable of producing apoptosis in the treated cells (Figure 6d). However, media taken 24 h.p.i. (and containing HMGB1) actually reduced the levels of apoptosis relative to background, implying that autophagy or necrosis is being induced instead.

\section{DISCUSSION}

The very real need for novel therapies of cancer has led to the development of a variety of biological approaches. One of which, the use of oncolytic viruses, has made significant progress in both pre-clinical and clinical development over the last 5 years. ${ }^{2,3}$ As these therapies move into later stage clinical testing, and eventual approval, it is almost certain they will be combined with other therapies to maximize their effectiveness. A detailed understanding of which oncolytic viruses combine most effectively with traditional therapies, and the mechanisms underlying these effects are therefore needed.

To date, the majority of this work has concentrated on how virusinfected cancer cells can either be destroyed more effectively, or become more susceptible to viral replication in the presence of chemo or radiotherapy. Although important, it can be noted that (i) oncolytic viruses will only ever be able to infect a portion of the cells within a tumor, and so a bystander effect (whereby neighboring, uninfected cells are also destroyed, or become more sensitive to other therapies) will be critical and (ii) because infected cells will be destroyed as a result of infection (either through apoptosis, viral mediated destruction or immune clearance) it is again important to examine the combination effects in both infected and uninfected cells.

We concentrated on the effects of combining paclitaxel with oncolytic vaccinia virus in a variety of different tumor models, including mouse and human, ovarian, lung, breast and colorectal cancers. We initially ran isobolagram studies following addition of the two therapies $24 \mathrm{~h}$ apart, and in different orders, and found that under all conditions tested synergy occurred. The fact that synergy occurred in all cell lines implied that common pathways may be mediating these effects, and that different mechanisms may mediate the effect depending on which order the agents were added. Although it is difficult to prove synergy in vivo, we did demonstrate that combination therapy resulted in significant therapeutic benefits in a mouse tumor model, while no additional toxicities were noted as a result of combining the therapies in vivo.

However, one concern with increasing tumor cell killing in the context of an oncolytic viral therapy is that viral replication may be prematurely curtailed (if infected cells are destroyed before replication can be completed). This was indeed found to be the case in vitro, with reduced viral gene expression and replication as a result of addition of paclitaxel. However, vaccinia has a complex life cycle, with several 
forms of the virus produced, including an EV form that is the first formed and secreted from infected cells early in the replication cycle. The non-enveloped form (MV) is not released until after cell lysis. Therefore, although the EV only represents a small percentage of the virus produced subsequent to infection of a susceptible cell, it is likely to be the most important for local and systemic spread within the host. Also, because it is produced early (in the first 6-18h) after infection we examined whether it might be produced before chemotherapy-mediated cell killing occurred. It was confirmed that despite more than a log reduction in total levels of virus produced when paclitaxel was added, there was no significant reduction in the levels of EV produced. The importance of this EV form during in vivo spread was seen with the fact that although a reduction in the viral gene expression from within the tumor was seen with paclitaxel combination in mouse studies, this was not significant.

Because it is known that vaccinia rapidly induces infected cells to enter S-phase after infection, ${ }^{18}$ and that this phase is also induced by paclitaxel treatment, we examined the possibility that the position of cells in the cell cycle may mediate synergy between these therapies, especially when the paclitaxel is added first. It was observed that not only can vaccinia induce entry into S-phase, but also the virus displayed a preferential infection of cells in this phase of the cell cycle. This is the first time such an effect has been reported, and it is perhaps unclear why this should be the case, as vaccinia does not have cognate receptors necessary for cell entry that may be specifically upregulated. However, vaccinia entry into target cells is thought to be mediated by heparin sulfate and other glucosaminoglycans (GAG) molecules on the cell surface, ${ }^{23,24}$ and several reports have indicated that the surface expression of these molecules are indeed upregulated during the S-phase of the cell cycle. ${ }^{25}$ This could therefore explain increased infection of cells in the S-phase. As a result early viral infection of tumor cells (within $2 \mathrm{~h}$ of exposure) was doubled when cells had been previously exposed to paclitaxel for $18 \mathrm{~h}$ and so were primarily blocked in S-phase.

We also observed that in our initial synergy studies when virus was added first, paclitaxel addition produced enhanced cell killing in wells with very low $(<0.01)$ MOIs of virus added. This indicated that viral infection might be producing a bystander effect with uninfected cells becoming sensitized to paclitaxel therapy simply due to their proximity to infected cells. This was tested with filter-sterilized media taken from infected cell layers at different times post-infection $(6,12$ and $24 \mathrm{~h}$ ) and adding this to uninfected paclitaxel treated cells. It was found that the media from infected cells alone was sufficient to enhance the sensitivity of a fresh cell layer to paclitaxel therapy. This is the first time that such a bystander effect produced by infected cancer cells has been observed. This was seen for a variety of different tumor cell lines, however some differences were observed. Primarily, it was seen that media taken $6 \mathrm{~h}$ post-infection ( 6 h.p.i.) from some, but not all cells was capable of enhancing sensitivity to paclitaxel primarily at low concentrations of paclitaxel. However, media taken 24 h.p.i. was universally capable of sensitizing cells over a wide range of paclitaxel concentrations. In addition, for at least some cells, media taken at 12 h.p.i. was not capable of inducing sensitivity, whereas that taken 6 and 24 h.p.i. was. Taken together, we hypothesized that this indicated at least two separate factors or combinations of factors released from infected cells (one that peaked around 6 h.p.i., and one at 24 h.p.i.) were each capable of sensitizing uninfected cells to paclitaxel therapy.

Because the factor(s) released at 6 h.p.i. must be produced before cell death, we focused on the possible production of cytokines from the infected cells. The primary mediator of the innate immune response released by epithelial cells is type I IFN, ${ }^{26}$ and so we initially focused on this cytokine as a candidate factor. It was seen that recombinant type I IFN could indeed sensitize cells to chemotherapy (an observation that has independently led to clinical trials involving the combination of these therapies ${ }^{27}$ ), and that infected cells were indeed capable of producing IFN. Finally, the use of anti-IFN receptor antibody to prevent activity of IFN from the 6 h.p.i. collected media verified that this cytokine was essential for sensitizing cells to paclitaxel at this time point. Because of the known therapeutic action of type I IFN, it is possible that this release of IFN may in itself represent a further cause for overall therapeutic benefit. Although other reports have demonstrated that recombinant IFN and paclitaxel can be used effectively in combination, this is the first time that release of IFN from oncolytic virus-infected cells has been shown to produce this same effect. The localized IFN production within selectively infected tumor cells may make this approach particularly attractive, as systemic toxicities would be avoided. In addition, a variety of oncolytic virus strains (including vaccinia) have been constructed that express IFN as a transgene, and that may prove particularly effective in this combination. ${ }^{28}$ Vaccinia expresses a type I IFN binding protein (B18R) that is know to sequester IFN released from infected cells, and that was expressed in the virus used in this work. ${ }^{29}$ However, no differences were seen when a B18R deleted virus strain was used (Supplemental Figure 3). It is unclear why this protein was unable to inhibit the IFN produced in these experiments, possibly the IFN was produced before the B18R expression, or at levels that overcame the B18R effects. B18R removal of IFN may explain why media taken at 12 h.p.i. did not typically mediate any sensitizing to paclitaxel exposure.

It was also observed that media taken at 24 h.p.i. was capable of sensitizing cells to paclitaxel, through what appeared to be an independent mechanism. It is likely that cells will begin to lyse as a direct result of viral infection by this time, and so DAMP release was hypothesized to be a possible mechanism that mediated this effect. ${ }^{30}$ HMGB1 is perhaps the best-characterized DAMP molecule, ${ }^{31}$ and so we examined whether this was released. It was demonstrated (for the first time) that vaccinia induced cell lysis was indeed capable of leading to HMGB1 release. Furthermore, the effect of an inhibitor of DAMP action (recombinant DAMP A-box) confirmed the importance of this molecule in sensitizing surrounding cells to paclitaxel. The addition of the HMGB1 inhibitor reduced the ability of media collected 24 h.p.i. to sensitize cells to paclitaxel. Because HMGB1 can induce autophagy, necrosis or apoptosis in exposed cells depending on its oxidative state and the presence of secondary factors, we examined the levels of apoptosis in cells under different conditions. It was found that media taken 24 h.p.i. actually reduced apoptosis levels. This could indicate that either autophagy or possibly necrosis is occurring in these cells.

It is not clear why HMGB1 release, that is typically associated with tumor progression and resistance to chemotherapy, ${ }^{32}$ might be sensitizing cells to paclitaxel treatment in this situation. It is possible that this acute release of HMGB1 (as opposed to the chronic release typically seen in the tumor environment) might be mediating a more beneficial response. ${ }^{33}$ For example, HMGB1 induced autophagy has been shown to sensitize cells to apoptosis in some situations. ${ }^{34}$ It is also possible that secondary factors released into the media, such as virally encoded proteins, or even viral DNA may be mediating increased sensitivity in combination with HMGB1 (HMGB1 and microbial DNA can interact to activate TLR9 signaling). Finally, it is possible that the proliferation inducing effects of HMGB1 may make the cells better targets for the cell cycle targeting paclitaxel therapy. These possibilities are currently being examined further.

We have therefore not only demonstrated that oncolytic vaccinia and paclitaxel can synergize in their cancer cell killing, but also have 
indicated several novel mechanisms that mediate this effect. These data may be incorporated into the design of more effective clinical trials and into the development of next generation therapies designed to enhance these interactions. In addition, these individual approaches to enhance the effectiveness of paclitaxel may be specifically developed.

\section{MATERIALS AND METHODS}

Cell lines and vaccinia strains

Human colorectal and breast cancer cell lines HCT-116 and MCF-7 were obtained from ATCC, the human ovarian cancer cell line UCI-101 was provided by Drs P DiSaia and A Manetta, University of California, San Diego, CA, USA, murine lung cancer cell line Lewis lung carcinoma was obtained from ATCC, Manassas, VA, USA, and the mouse colorectal cancer cell line CMT-93 was obtained from Cancer Research, London, UK. All cells were labeled to express luciferase through retroviral transfection. The vaccinia strain WR was obtained from ATCC, while the oncolytic strain vvDD is a version of WR with gene deletions in the viral growth factor and thymidine kinase gene and has been described previously. ${ }^{35}$ The WR strain containing an A5L-GFP fusion was provided by Geoff Smith (Imperial College, London, UK). ${ }^{20}$

\section{Cell killing and isobologram studies}

Cells were plated in 96-well plates to $80 \%$ confluency and were infected with serial dilutions of virus or paclitaxel (Taxol; Sigma-Aldrich, St Louis, MO, USA) as described previously, ${ }^{36}$ followed $24 \mathrm{~h}$ later by addition of the other therapy. Cell survival was determined $72 \mathrm{~h}$ later by MTS assay (Promega, Madison, WI, USA). Dose response curves were generated and concentrations killing $50 \%$ of the cells (EC50-values) determined relative to untreated cells. Four dilution rations were included in each test (virus/paclitaxel ratios of 3, 33, 333 and 3333; NB: each well contained 1000 cells and $200 \mu \mathrm{l}$ media). Isobolograms were generated and combination indexes established to define synergistic or antagonistic effects on cell death. Each data point was generated from triplicate samples and repeated three times.

\section{Mouse model}

HCT-116 tumor cells $\left(1 \times 10^{7}\right.$ per mouse) were implanted subcutaneously into athymic nu-/nu- mice. Once tumors formed $\left(50-100 \mathrm{~mm}^{3}\right)$ mice were treated with (i) $1 \times 10^{7}$ PFU of vvDD-luc delivered intravenously on day 1 ; (ii) paclitaxel $\left(60 \mathrm{mg} \mathrm{kg}^{-1}\right)$ delivered intraperitoneal on days 1,3 and 5 or both or phosphate-buffered saline controls. Tumor burden was followed by caliper measurement, viral biodistribution and persistence was followed by bioluminescence imaging (following luciferin injection and $2 \%$ isoflurane anesthesia, using an IVIS200 system). No overt signs of toxicity were observed. All animal work was performed with the approval from the Institutional Animal Care and Use Committee.

\section{Viral replication assays}

vvDD-luc was used to infect cells at an MOI of 2.0, subsequent viral gene expression was determined by bioluminescence imaging after addition of luciferin, overall replication was determined by plaque assay on BSC-1 cells, whereas EV production was determined by plaque assay of supernatant following addition of anti-MV neutralizing antibody (BEI Resources, Manassas, VA, USA)

\section{Flow cytometry assays}

Cells were infected with A5L-GFP virus and collected and stained with 7-AAD. Standard paramaters to determine cell cycle of the stained cells were used in a FACScaliber (BD Pharmingen, San Diego, CA, USA). Cell cycle status of GFP positive (virus infected) was also examined. In some experiments cells were treated with vincristine (Sigma).

\section{Preparation of media collected from virus infected cells}

Cell lines were infected in T-25 flasks at an MOI of 5.0 with vvDD. At times after infection, media was collected and filter sterilized through $0.1 \mu \mathrm{m}$ Inorganic membrane filters (Whatman International LTD, Maidstone, England) to remove any virus or floating cells in the supernatant (in some experiments complete removal of virus was verified by plaque assay). Medium collected in parallel from uninfected flasks was used as a control. In some experiments, IFN- $\beta$ levels in the collected media were measured by sandwich immunoassay ELISA (Verikine human IFN- $\beta$ ELISA kit, PBL Interferon Source, Piscataway, NJ, USA, or mouse IFN- $\beta$ ELISA, Biosource, Invitrogen, Carlsbad, CA, USA) according to manufacturer's instructions. In other experiments, HMGB1 levels were determined by sandwich ELISA (HMGB1 ELISA kit II, Shino-Test Corporation, Kanagawa, Japan). The experimental procedure was in accordance with the protocol supplied with the kit.

Assays to determine sensitivity of cells to paclitaxel chemotherapy Cells expressing luciferase were plated in dark wall 96 -well plates and $80 \mu \mathrm{l}$ per well of spent media from infected or uninfected cells added. At the same time, a dilution series of paclitaxel was added and the cells incubated for 24 or $48 \mathrm{~h}$ before cell viability was determined by both bioluminescenmce assay and MTS assay. In some experiments 250 or $500 \mathrm{U} \mathrm{ml}^{-1}$ of recombinant human or mouse type I IFN was added to the cells (Human or mouse leukocyte interferon, PBL Biomedical Laboratories, Piscataway, NJ, USA), whereas in other experiments, typel hIFN receptor blocking antibody (Mouse anti-human IFN $\alpha / \beta$ receptor chain 2 MAb, Millipore Corporation, Billerica, MA, USA) was also added into the wells $\left(0.5 \mu \mathrm{g} \mathrm{ml}^{-1}\right)$. Finally, in some experiments HMGB1 A-box peptide (provided by Dr Michael Lotze) was added at a final concentration of $300 \mathrm{ng} \mathrm{ml}^{-1}$, or the HMGB1 inhibitor glycyrrhizin added at $200 \mu \mathrm{m}$.

\section{Caspase assay}

UCI-101 cells were treated with Taxol $\left(6 \mathrm{ng} \mathrm{ml}^{-1}\right)$ or media collected $6 \mathrm{~h}$ or $24 \mathrm{~h}$ post infection, or combinations of these two. After $24 \mathrm{~h}$ cells were collected and lysed. The cell lysates were used to perform a caspase assay (ApoAlert caspase assay plates, Clontech Laboratories, Inc., Mountain View, CA, USA). The experimental procedure was in accordance with the protocol supplied with the kit. The plate was read in a plate reader with $380 \mathrm{~nm}$ excitation and $460 \mathrm{~nm}$ emission filter.

\section{Statistical analysis}

Groups were compared using 2-tailed Student's $t$-test (having confirmed normal distribution of cohorts by Kolmogorov-Smirnov test), other than survival curves that were compared by Wilcoxon analyses. A $P$-value $<0.05$ was considered significant.

\section{CONFLICT OF INTEREST}

Dr David Kirn is CEO of Jennerex Biotherapuetics.

\section{ACKNOWLEDGEMENTS}

We thank Dr Geoff Smith (Imperial College, London, UK) for the A5L-GFP virus and Dr David Bartlett (University of Pittsburgh, Pittsburgh, PA, USA) for vvDD. This work was supported by the Hillman Fellowship.

1 Guo ZS, Thorne SH, Bartlett DL. Oncolytic virotherapy: molecular targets in tumor-selective replication and carrier cell-mediated delivery of oncolytic viruses. Biochim Biophys Acta 2008; 1785: 217-231. Epub 15 Feb 2008.

2 Kirn DH, Thorne SH. Targeted and armed oncolytic poxviruses: a novel multimechanistic therapeutic class for cancer. Nat Rev Cancer 2009; 9: 64-71.

3 Park BH, Hwang T, Liu TC, Sze DY, Kim JS, Kwon HC et al. Use of a targeted oncolytic poxvirus, JX-594, in patients with refractory primary or metastatic liver cancer: a phase I trial. Lancet Oncol 2008; 9: 533-542.

4 Vidal L, Pandha HS, Yap TA, White CL, Twigger K, Vile RG et al. A phase I study of intravenous oncolytic reovirus type 3 Dearing in patients with advanced cancer. Clin Cancer Res 2008; 14: 7127-7137.

5 Galanis E, Hartmann LC, Cliby WA, Long HJ, Peethambaram PP, Barrette BA et al. Phase I trial of intraperitoneal administration of an oncolytic measles virus strain engineered to express carcinoembryonic antigen for recurrent ovarian cancer. Cancer Res 2010; 70: 875-882.

6 Senzer NN, Kaufman HL, Amatruda T, Nemunaitis M, Reid T, Daniels G et al. Phase II clinical trial of a granulocyte-macrophage colony-stimulating factor-encoding, secondgeneration oncolytic herpesvirus in patients with unresectable metastatic melanoma. J Clin Oncol 2009; 27: 5763-5771. 
7 Kumar S, Gao L, Yeagy B, Reid T. Virus combinations and chemotherapy for the treatment of human cancers. Curr Opin Mol Ther 2008; 10: 371-379.

8 Harrington KJ, Melcher A, Vassaux G, Pandha HS, Vile RG. Exploiting synergies between radiation and oncolytic viruses. Curr Opin Mol Ther 2008; 10: 362-370.

9 Nguyen TL, Tumilasci VF, Singhroy D, Arguello M, Hiscott J. The emergence of combinatorial strategies in the development of RNA oncolytic virus therapies. Cell Microbiol 2009; 11: 889-897.

10 Galanis E, Okuno SH, Nascimento AG, Lewis BD, Lee RA, Oliveira AM et al. Phase I-II trial of ONYX-015 in combination with MAP chemotherapy in patients with advanced sarcomas. Gene Ther 2005; 12: 437-445.

11 Lamont JP, Nemunaitis J, Kuhn JA, Landers SA, McCarty TM. A prospective phase II trial of ONYX-015 adenovirus and chemotherapy in recurrent squamous cell carcinoma of the head and neck (the Baylor experience). Ann Surg Oncol 2000; 7: 588-592.

12 Gutermann A, Mayer E, von Dehn-Rothfelser K, Breidenstein C, Weber M, Muench M et al. Efficacy of oncolytic herpesvirus NV1020 can be enhanced by combination with chemotherapeutics in colon carcinoma cells. Hum Gene Ther 2006; 17: 1241-1253.

13 Liu C, Sarkaria JN, Petell CA, Paraskevakou G, Zollman PJ, Schroeder M et al. Combination of measles virus virotherapy and radiation therapy has synergistic activity in the treatment of glioblastoma multiforme. Clin Cancer Res 2007; 13: 7155-7165.

14 AbouEl Hassan MA, Braam SR, Kruyt FA. Paclitaxel and vincristine potentiate adenoviral oncolysis that is associated with cell cycle and apoptosis modulation, whereas they differentially affect the viral life cycle in non-small-cell lung cancer cells. Cancer Gene Ther 2006; 13: 1105-1114.

15 Passer BJ, Castelo-Branco P, Buhrman JS, Varghese S, Rabkin SD, Martuza RL. Oncolytic herpes simplex virus vectors and taxanes synergize to promote killing of prostate cancer cells. Cancer Gene Ther 2009; 16: 551-560.

16 Nagano S, Perentes JY, Jain RK, Boucher Y. Cancer cell death enhances the penetration and efficacy of oncolytic herpes simplex virus in tumors. Cancer Res 2008; 68: 3795-3802.

17 Smith GL, Vanderplasschen A, Law M. The formation and function of extracellular enveloped vaccinia virus. J Gen Virol 2002; 83: 2615-2631.

18 Wali A, Strayer DS. Infection with vaccinia virus alters regulation of cell cycle progression. DNA Cell Biol 1999; 18: 837-843.

19 Long BH, Fairchild CR. Paclitaxel inhibits progression of mitotic cells to G1 phase by interference with spindle formation without affecting other microtubule functions during anaphase and telephase. Cancer Res 1994; 54: 4355-4361.

20 Carter GC, Rodger G, Murphy BJ, Law M, Krauss O, Hollinshead M et al. Vaccinia virus cores are transported on microtubules. J Gen Virol 2003; 84: 2443-2458.

21 Yang H, Tracey KJ. Targeting HMGB1 in inflammation. Biochim Biophys Acta 2010; 1799: 149-156
22 Yang H, Ochani M, Li J, Qiang X, Tanovic M, Harris HE et al. Reversing established sepsis with antagonists of endogenous high-mobility group box 1 . Proc Natl Acad Sci USA 2004; 101: 296-301.

23 Chung CS, Hsiao JC, Chang YS, Chang W. A27L protein mediates vaccinia virus interaction with cell surface heparan sulfate. J Virol 1998; 72: 1577-1585.

24 Hsiao JC, Chung CS, Chang W. Cell surface proteoglycans are necessary for A27 L protein-mediated cell fusion: identification of the $\mathrm{N}$-terminal region of $\mathrm{A} 27 \mathrm{~L}$ protein as the glycosaminoglycan-binding domain. J Virol 1998; 72: 8374-8379.

25 Moreira CR, Lopes CC, Cuccovia IM, Porcionatto MA, Dietrich CP, Nader HB. Heparan sulfate and control of endothelial cell proliferation: increased synthesis during the $\mathrm{S}$ phase of the cell cycle and inhibition of thymidine incorporation induced by ortho-nitrophenyl-beta-D-xylose. Biochim Biophys Acta 2004; 1673: 178-185.

26 Tough DF. Type I interferon as a link between innate and adaptive immunity through dendritic cell stimulation. Leuk Lymphoma 2004; 45: 257-264.

27 Schneider B, Fukunaga A, Murry D, Yoder C, Fife K, Foster A et al. A phase I, pharmacokinetic and pharmacodynamic dose escalation trial of weekly paclitaxel with interferon-alpha2b in patients with solid tumors. Cancer Chemother Pharmacol 2007; 59: 261-268.

28 Kirn DH, Wang Y, Le Boeuf F, Bell J, Thorne SH. Targeting of interferon-beta to produce a specific, multi-mechanistic oncolytic vaccinia virus. PLoS Med 2007; 4: e353.

29 Symons JA, Alcami A, Smith GL. Vaccinia virus encodes a soluble type I interferon receptor of novel structure and broad species specificity. Cell 1995; 81: 551-560.

30 Garg AD, Nowis D, Golab J, Vandenabeele P, Krysko DV, Agostinis P. Immunogenic cell death, DAMPs and anticancer therapeutics: an emerging amalgamation. Biochim Biophys Acta 2010; 1805: 53-71.

31 Lotze MT, Tracey KJ. High-mobility group box 1 protein (HMGB1): nuclear weapon in the immune arsenal. Nat Rev Immunol 2005; 5: 331-342.

32 Tang D, Kang R, Zeh 3rd HJ, Lotze MT. High-mobility group box 1 and cancer. Biochim Biophys Acta 2010; 1799: 131-140.

33 Campana L, Bosurgi L, Rovere-Querini P. HMGB1: a two-headed signal regulating tumor progression and immunity. Curr Opin Immunol 2008; 20: 518-523.

34 Zhuang W, Qin Z, Liang Z. The role of autophagy in sensitizing malignant glioma cells to radiation therapy. Acta Biochim Biophys Sin (Shanghai) 2009; 41: 341-351.

35 McCart JA, Ward JM, Lee J, Hu Y, Alexander HR, Libutti SK et al. Systemic cancer therapy with a tumor-selective vaccinia virus mutant lacking thymidine kinase and vaccinia growth factor genes. Cancer Res 2001; 61: 8751-8757.

36 Cheong SC, Wang Y, Meng JH, Hill R, Sweeney K, Kirn D et al. E1A-expressing adenoviral $\mathrm{E} 3 \mathrm{~B}$ mutants act synergistically with chemotherapeutics in immunocompetent tumor models. Cancer Gene Ther 2008; 15: 40-50.

Supplementary Information accompanies the paper on Gene Therapy website (http://www.nature.com/gt) 